\title{
e-Phaïstos
}

e-Phaïstos

Revue d'histoire des techniques / Journal of the history

of technology

V-2 | 2016

Histoire des techniques en Afrique de l'Ouest

\section{Mutations techniques dans le domaine de la fonderie à Kolonkani-Ba (Province de la Kossi, Burkina Fasso)}

Technical Changes in the Field of Foundry in Kolonkani-Ba (province of

Kossi,Burkina Faso)

Élise-Foniyama Thiombiano-Ilboudo

\section{(2) OpenEdition}

\section{Journals}

Édition électronique

URL : http://journals.openedition.org/ephaistos/5867

DOI : 10.4000/ephaistos.5867

ISSN : 2552-0741

Éditeur

IHMC - Institut d'histoire moderne et contemporaine (UMR 8066)

Édition imprimée

Date de publication : 15 juin 2016

Pagination : $92-102$

ISSN : 2262-7340

Référence électronique

Élise-Foniyama Thiombiano-Ilboudo, « Mutations techniques dans le domaine de la fonderie à Kolonkani-Ba (Province de la Kossi, Burkina Fasso) », e-Phaïstos [En ligne], V-2 | 2016, mis en ligne le 15 mars 2020, consulté le 18 juin 2020. URL : http://journals.openedition.org/ephaistos/5867 ; DOI https://doi.org/10.4000/ephaistos.5867 


\section{Mutations techniques dans le domaine de la fonderie à Kolonkani-Ba (Province de la Kossi, Burkina Fasso)}

\begin{abstract}
Introduction
«L'histoire des techniques ne peut cependant pas se faire en dehors de la société, de la culture et des pensées qui constituent un ensemble avec la technique à proprement parler. En effet, la technique qui est la particularité qu'a l'homme à inventer les outils, les procédés pour agir sur son environnement de façon durable et reproductible est le plus souvent le reflet de la culture, de la société et de la pensée, en un mot de l'homo technicus dans son environnement. $»^{1}$
\end{abstract}

L'histoire des techniques, de la pensée technique est avant tout l'histoire de la société, de la culture et des hommes. C'est dans ce cadre que nous plaçons le cas d'étude de l'invention de Adama Traoré, fondeur du village de Kolonkani-Ba.

Le village de Kolonkani-Ba se localise dans la commune de Kombori, qui fait partie de la province de la Kossi (cf. carte). Il est habité par des populations Dafing, Bobo et Dioula. Le père

\author{
Elise Foniyama Thiombiano \\ Ilboudo \\ Maître assistant, UFR/SH, département \\ d'histoire et d'archéologie \\ Université de Ouagadougou \\ Burkina Fasso
}

d'Adama Traoré avait fait un séjour de 40 ans à la Mecque où il se rendit à pieds depuis le Burkina Faso, avec sa femme. Au cours de son voyage aller, comme au retour, il passa des années dans des pays comme le Mali, la Mauritanie, le Niger et le Soudan².

Pour survivre, il menait des activités agricoles dans les régions qu'il traversait et les récoltes lui servaient pendant son parcours pour se nourrir. Sur le chemin de retour, il s'installa quelque temps au Soudan avec sa femme où tous deux apprirent la fabrication des marmites en aluminium auprès de maîtres soudanais. Adama Traoré naquit au Soudan et fut initié au métier de ses parents. De retour au Burkina Faso, il inventa un mécanisme pour un meilleur rendement de la production énergétique en utilisant l'énergie solaire et un système de conduit original pour la soufflerie du foyer. Au Burkina Faso, l'origine de l'un des groupes de fondeurs a pour origine le Mali, précisément Ségou et Hombori. Le village de Kolonkani-Ba se situe à la frontière du Mali et dans une région de fondeurs.

Pour cette étude, nous avons eu recours aux sources orales, écrites ainsi qu'à l'expérimentation. 


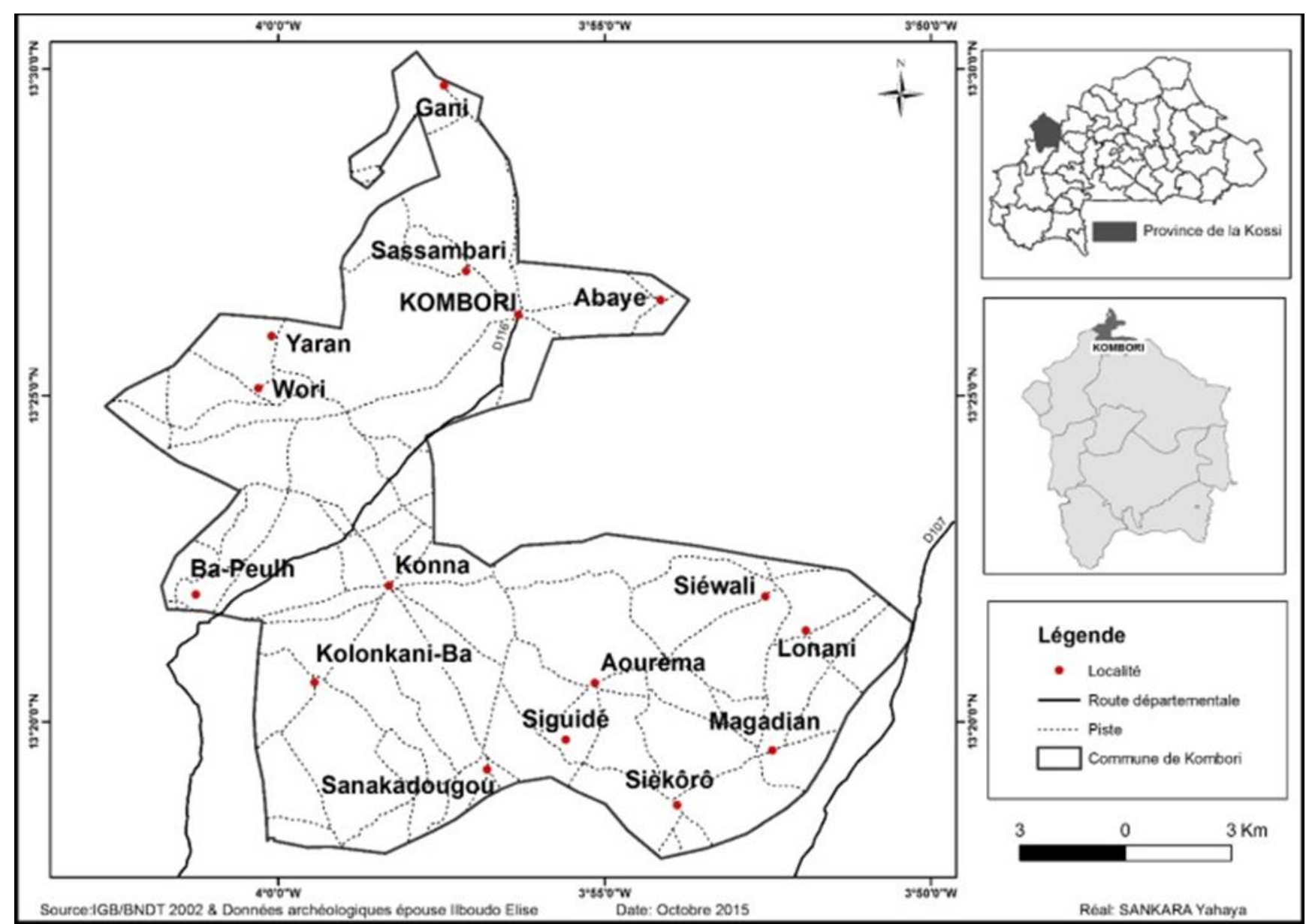

Figure 1 : Localisation du village de KOLONKANI-BA.

Présentation de l'atelier, de son équipement et de la matière première

Les sociétés se construisent à travers leurs techniques. C'est en ce sens que Anne-Françoise Garçon et al. affirment :

«Sans techniques, en effet, il n'y aurait ni patrimoine matériel et immatériel de la production, ni même de patrimoine culturel ou de paysage anthropique $» 3$.

Une des techniques nouvellement connues en Afrique de l'Ouest est la fonte à la cire perdue, mentionnée par Danilo Grebenart au début du XI ${ }^{\mathrm{e}}$ siècle à Toguéré Doupwili, dans l'actuel Mali4. Aujourd'hui, les techniques évoluent et celle mise au point par Adama Traoré vise à plus de rendement énergétique. Ici, la cire n'est pas utilisée mais les 
procédés permettent toutefois d'obtenir des résultats intéressants. À Kolonkani-Ba, pour la production des marmites en aluminium, le fondeur dispose d'un atelier, de matériels d'équipement et de la matière première.
Foyer

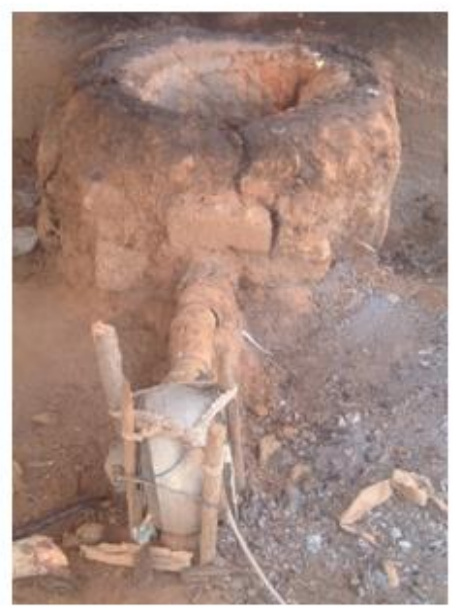

Bobine reliée au foyer par un conduit

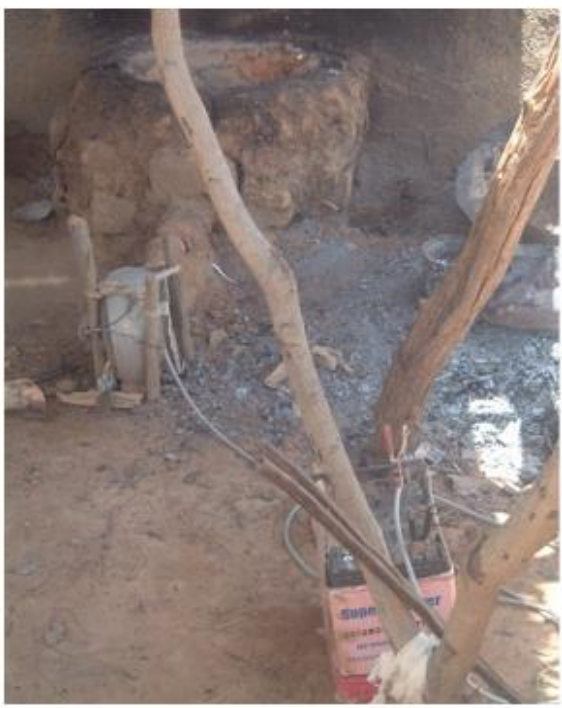

Bobine électrique

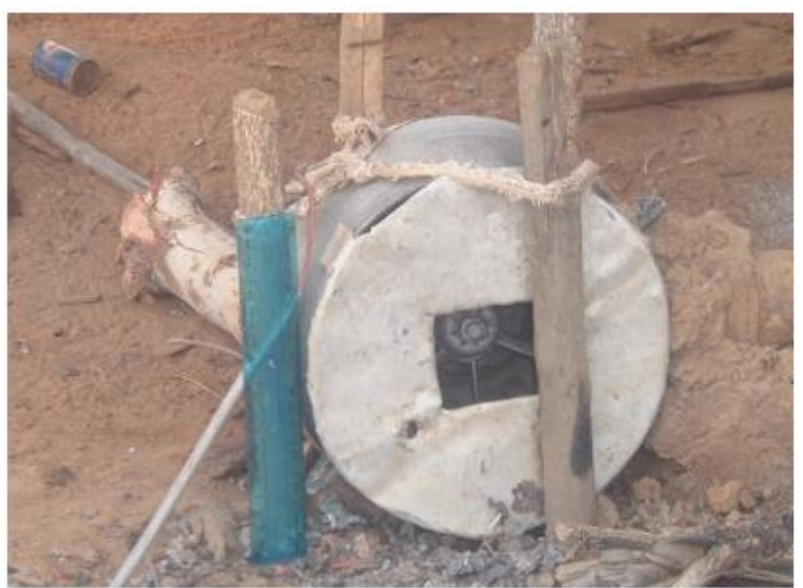

Plaques solaires reliées à une batterie

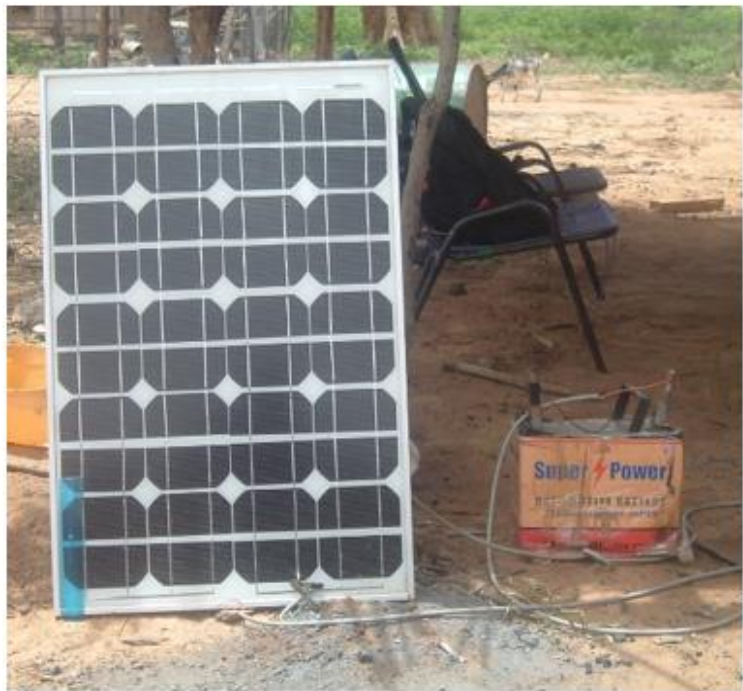

Figure 2 : vue du matériel de production des marmites en aluminium. 
Les ateliers de forge ou de fonte sont variables d'une région à une autre mais aussi en fonction des forgerons ou des fondeurs et des techniques de production. Ici, l'atelier de travail est constitué de poteaux implantés au sol et adossé au mur de la concession. Ailleurs on rencontre des ateliers similaires. Notamment, Zakaria Lingani, dans son écrit consacré à la production du bronze à Ouagadougou signale que

$$
\text { « l'atelier traditionnel est un }
$$
ensemble de lieux dans l'enceinte domestique ». Il ajoute que « c'est un hangar aménagé à l'extérieur de la concession avec un toit de paille posé sur quelques poteaux $» 5$.

En effet, à Konlonkani-ba, l'atelier est en-dehors de la concession et le toit est couvert de paille, audessus de laquelle se trouve une bâche en plastique qui protège les travailleurs. Cependant, il est ouvert de tous les côtés, ce qui en facilite l'accès. Aussi, une partie de l'équipement est exposé hors de l'atelier dont l'espace est bien aménagé.

Dans l'atelier, on distingue le foyer. Il est construit sans fondation sur le sol : deux couches de briques en terre battue sont déposées directement sur le sol. La structure a une forme circulaire. L'ouverture a un diamètre de $40 \mathrm{~cm}$ interne et $60 \mathrm{~cm}$ externe environ. L'épaisseur de la paroi, qui est d'environ $20 \mathrm{~cm}$, vise la conservation de hautes températures pour le travail car il s'agit là de la fonderie. Les briques sont reliées entre elles par des mottes de terre. Le foyer dispose d'une ouverture de $8 \mathrm{~cm}$ de diamètre dans le mur. Ce dernier reçoit un fer circulaire de $15 \mathrm{~cm}$ de long qui, relié à un tuyau, collecte l'air pour le foyer. L'équipement de l'atelier de la fonderie est variable.

Parmi le matériel disposé dans la fabrique se trouve aussi une bobine électrique. Par définition,

« la bobine auto-inductance est un composant courant en électrotechnique et électronique qui est constitué d'un enroulement de fils conducteurs éventuellement autour d'un noyau en matériau ferro-magnétique qui peut être un assemblage de feuille de tôles ou un bloc de ferrites (céramique ferro magnétique ${ }^{6}$.

Dans la fonderie d'Adama Traoré, la bobine électrique est un matériel de récupération de vielles voitures servant à l'origine au refroidissement du moteur des véhicules. Elle joue un rôle important dans le système de fonderie mis en place à Kolonkani-Ba. Il s'agit d'une pièce capitale qui intervient dans le système de production de l'énergie et dans le fonctionnement de la production.

Dans l'atelier, des marmites en aluminium coupées en deux parts égales dans le sens vertical servent de modèles. Les deux morceaux de la marmite présentent chacun une coupe dans le fond constituant des demi-cercles. Cet orifice servira à recueillir le liquide pour prendre la forme du moule.

En plus, deux cadres en bois sont utilisés dans le processus de production. Le premier est formé de deux moitiés de cadres qui sont reliés pour former un moule carré. Le second cadre, plus petit que le premier, constitue un carré fermé.

Enfin, une grande quantité d'argile est entassée dans un coin de la fabrique car elle a aussi un rôle fondamental dans le processus de fabrication. L'argile est utilisée à plusieurs niveaux, pour stabiliser le moule mais aussi pour le bourrage du modèle. L'argile et le sable interviennent dans bien des cas de production de céramique et dans le processus de travail des métaux. En effet,

«L'argile intervient dans le volet céramique de l'art du bronze. C'est avec l'argile que le fondeur fabrique ses creusets, ses tuyères, son foyer, le moulage des figurines, les noyaux ect... $» 7$.

Pour ce qui est de l'équipement de l'atelier à Kolonkani-Ba, on compte aussi une batterie. Une batterie de 12 volts destinée à l'alimentation du circuit électrique des voitures est achetée pour le 


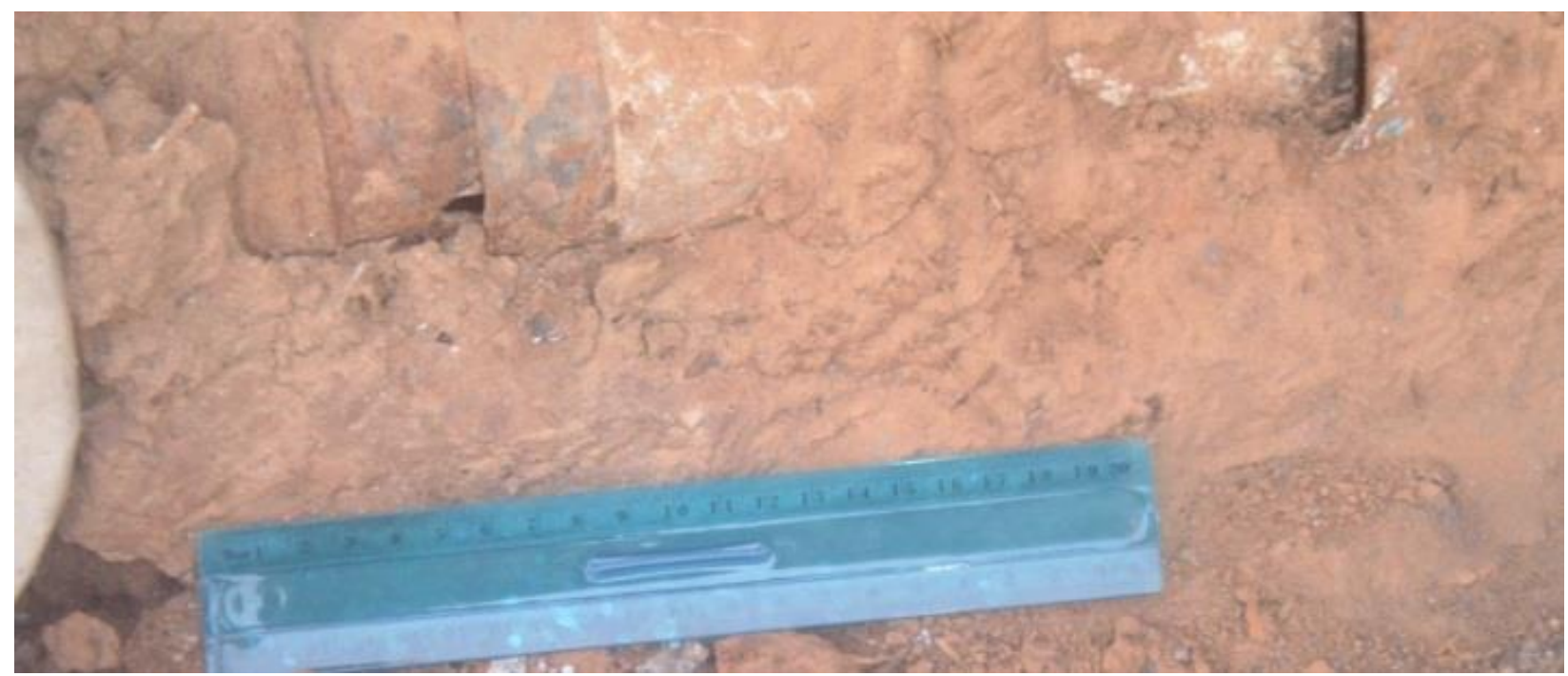

Figure 3 : conduit d'air fabriqué à partir d'anneaux en fer.

fonctionnement de la fonderie. Puis, des plaques solaires d'une hauteur de $70 \mathrm{~cm}$ et de $50 \mathrm{~cm}$ de large sont utilisées.

Un conduit d'air confectionné à partir d'anneaux métalliques ayant un diamètre d'environ $7 \mathrm{~cm}$ et une épaisseur de $2 \mathrm{~cm}$ ainsi que des fils électriques conducteurs de courant font partie du dispositif.

$\mathrm{Au}$ nombre des outils, on y trouve de longues pinces de $30 \mathrm{~cm}$ et un burin d'une longueur de 20 $\mathrm{cm}$.

Un récipient en fer est destiné à contenir les objets en aluminium à fondre. C'est le creuset, récipient en fonte utilisé pour liquéfier les métaux.

Tout comme nous l'indique Zakaria Lingani :

« le creuset est de forme conique à la base et circulaire dans sa partie supérieure» ${ }^{8}$.

Ici, la coupelle qui est utilisée dans le foyer a environ $20 \mathrm{~cm}$ de haut et $20 \mathrm{~cm}$ de diamètre. Tout l'équipement mentionné plus haut a pour objectif l'utilisation d'une matière première nécessaire à la fabrication des marmites.
Ce matériau a plusieurs origines. Il est obtenu à partir de matériel de récupération tels que des jantes de motos et de véhicules, des bobines de motos, de vielles marmites ou de vieux plats en aluminium, de vieilles boîtes vides de canettes de boisson et tout autre objet contenant de l'aluminium.

\section{Mécanisme de production des marmites en aluminium}

L'énergie est un élément capital pour faire fonctionner le système qui permet de produire les marmites en aluminium. À cet effet, Adama Traoré a eu une ingénieuse idée. À ce propos, Maclovia Correa de Silva indique que :

« Un axe de l'histoire des techniques se rapporte aux savoirs techniques et à ses acteurs. La culture et la transmission de ces savoirs à travers l'enseignement organisé, les pratiques du travail, la migration peuvent être un 
Creuset

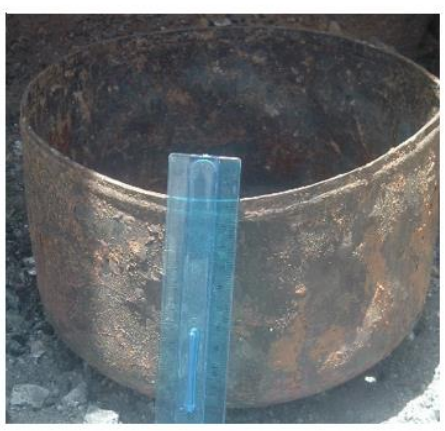

Fondeur martelant le sable autour du moule

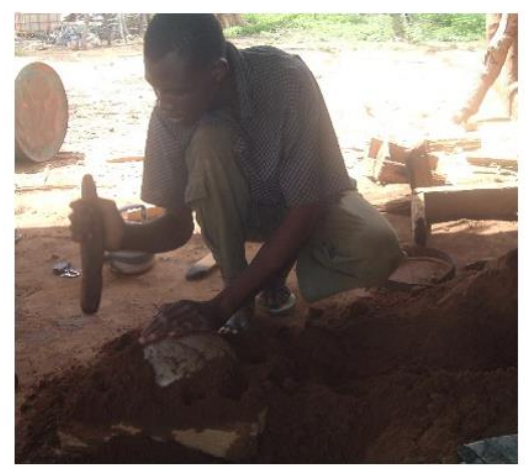

Cadre en bois et moule

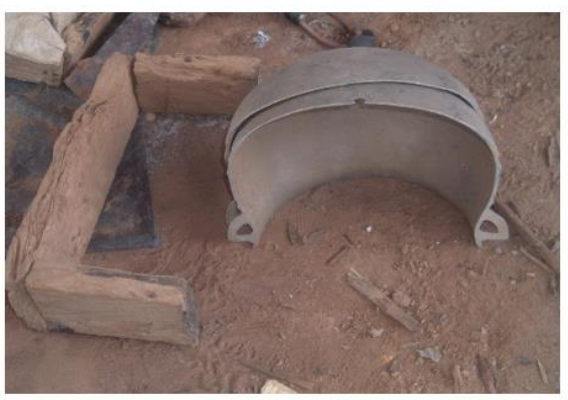

Moule dans le cadre

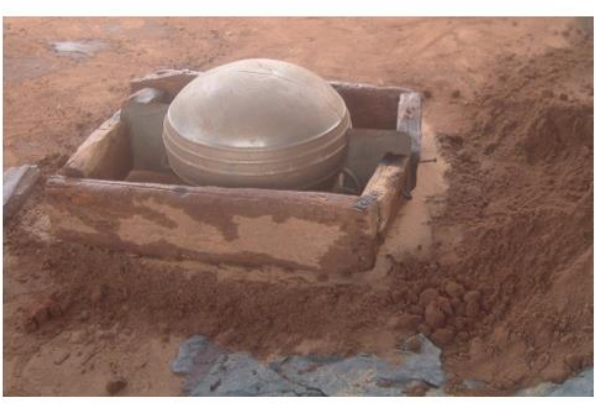

Marmite et couvercle en aluminium
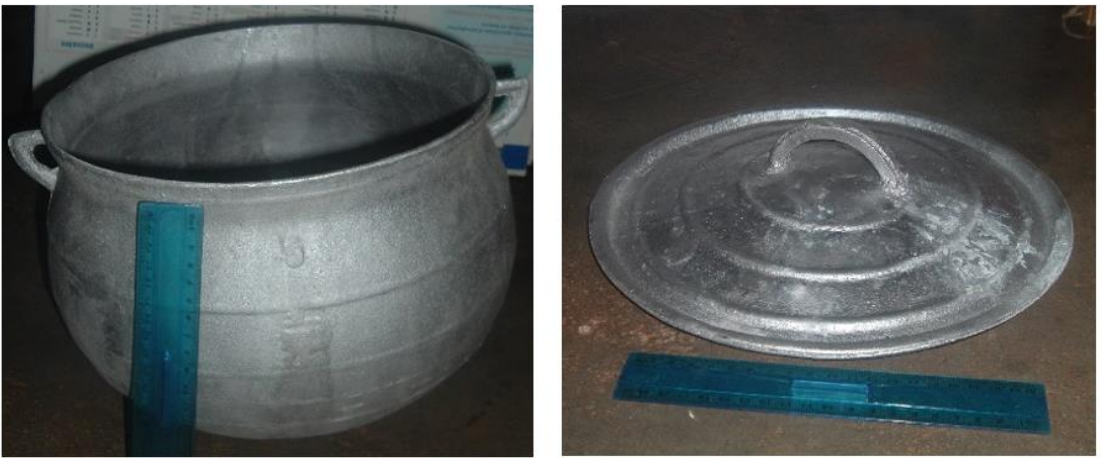

Figure 4: Vues du matériel de production de marmites en aluminium.

point de départ pour restituer l'usage des sources d'énergie de la planète et comment elles ont été pensées pour modifier les routines des villes. »9

Pour faire fonctionner le mécanisme, le fondeur a aménagé un endroit où sont disposées des plaques solaires au sol, orientées vers le soleil. Ces plaques peuvent être aussi placées en hauteur. Les plaques solaires reçoivent les rayons du soleil et produisent l'énergie utile à la fonderie. Elles sont reliées par des fils conducteurs à la batterie, placée à moins d'un mètre et en direction du foyer. L'énergie captée est envoyée dans l'accumulateur que constitue la batterie. Celle-ci se charge continuellement tant que la liaison n'est pas coupée. Un autre fil relie la batterie à la bobine. La bobine électrique est placée dans une marmite usagée avec la partie du ventilateur orientée vers l'ouverture. Cette marmite joue ici un rôle de protection de la bobine. Un couvercle en tôle percée au centre recouvre l'ouverture de la marmite. Ce trou permet l'aération de la bobine. La perforation faite dans le couvercle 
en tôle de la marmite permet également une ventilation. La marmite est couchée horizontalement de sorte à orienter la partie ouverte vers le sens opposé au foyer. Des piquets en bois servent à caler la marmite pour éviter que celle-ci ne bouge.

Le fondeur a imaginé une technique différente de l'ancienne pour produire les marmites. L'ancienne qui fonctionnait à l'aide d'une roue va évoluer vers l'utilisation d'un matériel nouveau. Céline Al-Zoubi, exprime cette évolution des techniques par l'affirmation selon laquelle :

«(...) ni culture, ni technique ne sont des entités figées mais des structures perméables dont le mouvement s'opère dans des temps et des lieux déterminés ${ }^{10}$.

Pour travailler, le fondeur établit la connexion entre la batterie et la bobine électrique. La connexion permet à la bobine de tourner et d'envoyer l'air dans le foyer. Le foyer et la bobine sont reliés par du tuyau confectionné à partir de plusieurs anneaux métalliques.

L'installation est renforcée avec de l'argile maçonnée tout au long du conduit horizontal. Le conduit est de forme conique. Cependant, la partie reliée directement au foyer est maçonnée sous forme rectangulaire. L'air est ainsi propulsé par la bobine dans le foyer dont l'alimentation entretient la flamme du charbon préalablement allumé. La soufflerie est permanente tant que la bobine n'est pas déconnectée de la batterie.

Disons avec Zakaria Lingani que pour l'opération de fonte et de moulage :

«les opérations débutent par la mise à feu du foyer et l'utilisation du creuset dans lequel le fondeur aura mis les quantités de métaux nécessaires à la coulée des moules prévus. " ${ }^{11}$

Les objets de récupération fondent doucement sous le contrôle du fondeur, qui remue de temps à autre le contenu du creuset. Après deux heures de cuisson environ, le métal bouillonne et devient au fur et à mesure liquide. Les impuretés, plus légères, remontent à la surface du liquide. Le fondeur les enlève au fur et à mesure et verse les saletés à côté du foyer.

Avant le coulage, un tapis d'argile d'une hauteur d'environ $5 \mathrm{~cm}$ est réalisé au sol. Son étendue tient compte des dimensions des cadres en bois. La tapisserie sert d'appui et permet la stabilité du moule. Les deux morceaux du modèle sont reliés pour former la marmite modèle. Le récipient est bourré de la même argile que celle ayant servi au tapis et placé au-dessus du carré d'argile, le fond orienté vers le haut. Un premier cadre constitué des deux parts sont reliés solidement à l'aide de fils métalliques et placés autour de la marmite. Mais avant cette opération, le cadre est aspergé d'eau dans le but d'empêcher l'argile d'adhérer au bois, ce qui facilite le décollage. Deux plaques métalliques très minces (quelques millimètres) chacune sont placées ensuite au niveau des jonctions de chaque côté du bas jusqu'à mi-hauteur de la marmite. Elles s'étendent des jonctions des marmités aux jonctions du cadre en bois qui l'entoure. Son emplacement aux extrémités facilite le démoulage. L'argile, bien mélangée avec de l'eau pour obtenir une consistance qui convienne, est également utilisée pour bourrer ce premier cadre. Une massue en fer est utilisée pour tasser le sable autour du modèle. Après cette étape, le fondeur s'assure que l'argile a pris la forme du modèle. Sur le premier cadre, un second est placé pour couvrir entièrement le modèle. Une fois de plus l'argile est tassée autour et au-dessus du modèle. Lorsque l'ensemble est bien compact, l'artisan retire le cadre du dessus. Ensuite, il sépare les deux cadres en bois bourrés également de sable grâce à la plaque métallique précédemment placée entre eux. Cette dernière est retirée délicatement et une boule d'argile est reconstituée au centre. Aussi, le modèle est retiré et les empreintes laissées par les deux morceaux du modèle sont rassemblés autour de la boule d'argile. 
Lorsque le retrait du moule est effectif, le forgeron reconstitue ainsi la forme laissée dans l'argile par la marmite modèle. Entre la boule et les deux cadres se trouve ainsi un creux interne destiné à accueillir le liquide d'aluminium. D'autres techniques de moulage ont été utilisées pour fabriquer des objets en bronze. Par exemple, pour Denise Paulme

«La fonte à la cire perdue était connue de l'Égypte ancienne, qui en transmit le secret à la civilisation gréconubienne de Méroé ».

Depuis des périodes très reculées, des techniques de moulage ont été utilisées. C'est dans ce cadre que Joseph Ki-Zerbo cite R. Cornevin :

« c'est probablement par les Arabes que la technique du moulage à la cire perdue pratiquée dans toute l'Eurasie dès l'âge du bronze traversa le Sahara $»^{12}$.

"Certains détails des pièces nilotiques du début de l'ère chrétienne ont leur pendant sur les bronzes d'Ifé ${ }^{13}$.

\section{Zakaria Lingani ajoute que}

« la métallurgie du cuivre et de ses alliages s'accompagne également des techniques aboutissant à la transformation du métal, principalement la technique de la fonte à la cire perdue ${ }^{14}$.

Après ce processus de création du creux, le fondeur passe au coulage. Le coulage est une opération à la fois rapide et délicate. Pour cette opération, il est muni des pinces destinées à saisir le creuset contenant l'aluminium fondu. Le liquide est vidé dans le creux du moule à l'aide d'un entonnoir. Il n'est pas toujours nécessaire d'utiliser un entonnoir car parfois, le creuset porte un bec verseur. Il prend alors la forme de l'empreinte laissé par celui-ci. L'aluminium se solidifie et épouse la forme. Un temps est observé pour permettre la consolidation.

Puis intervient le démoulage lorsque le fondeur juge la consolidation faite. Cette opération consiste à séparer la marmite du moule d'argile en le dégageant. En effet, une demi-heure après, l'argile est dégagée et la nouvelle marmite est retirée. Pour cela, le fondeur retire d'abord le moule placé audessus, puis celui du bas.

Une autre étape après le retrait du sable est celle $\mathrm{du}$ raffermissement de la marmite en dehors du moule. Pour cela, le fondeur utilise de l'eau froide qu'il verse sur le nouveau récipient pour une plus grande consolidation. Ainsi dégagée, la nouvelle marmite porte des irrégularités qu'il faut aussi nettoyer.

C'est l'étape de l'affinage ou la finition de l'œuvre. Pour Ibrahim Zoungrana, «c'est l'ensemble des procédés destinés à rendre la pièce prête à la vente. Elle consiste à ébarber, meuler, limer, souder, polir et ciseler la pièce sortie du moule pour faire disparaitre les évents et d'autres couches indésirées $\gg 15$.

L'orifice qui avait servi à faire couler le liquide dans le creux est rebouché et laisse sortir un rebondi. Le fondeur coupe d'abord la partie débordante et scie les aspérités du bord. Ensuite, l'ensemble subit le polissage et le lissage. Lorsque les rugosités sont éliminées, la marmite est prête pour aller sur le feu. Pour ce qui est des couvercles, le même procédé est utilisé. La différence se situe au niveau des moules.

Les avantages de l'usage de cette technique sont de plusieurs ordres. Avec ce mécanisme, l'inventeur affirme qu'il économise la moitié du temps qu'il perdait pour la production d'un ustensile en aluminium avec le tour. En plus du gain en temps, le personnel est considérablement réduit. Dans ce cadre, le travail est aussi moins salissant. Une seule 
personne suffit pour mettre le mécanisme en marche et produire un objet. Un autre avantage est l'économie de l'effort physique que le forgeron use en tournant la manivelle du tour en permanence pour alimenter le foyer en air. Aussi, les métaux atteignent le degré de fluidité nécessaire au moulage. En effet,

«le fondeur devrait veiller attentivement à la fonte en maintenant de façon continue la température du foyer car seul le point de fusion maximum donne à l'alliage la fluidité nécessaire permettant une meilleure prise de l'empreinte du moule ${ }^{16}$.

L'avantage de ce système est qu'il permet de maintenir le chauffage et la fusion tout le temps de production. C'est un processus permettant un meilleur rendement, car le fondeur produit plus de marmites avec ce mécanisme. La conséquence directe est que cela lui permet de vendre plus et de faire plus de bénéfices.

Parmi les inconvénients, les charges sont plus élevées à cause du coût d'investissement de départ

1 KABORE-KIENON Timpoko Hélène, 2013, L'imaginaire des techniques anciennes en Afrique subsaharienne ", Communication au colloque sur « L'imaginaire et les techniques » du 14 au 16 novembre 2013, Paris, France.

2 TRAORE Adama, enquête réalisée le 20 juillet 2015 à Kolonkani-Ba.

3 GARÇON Anne-Françoise et al., 2010, Introduction, pp. IXXVIII ; In Techniques, Patrimoine, Territoires de l'Industrie : Quel enseignement? International Workshop, 227 p., p. XI.

4 GREBENART D., 1988, Les premiers métallurgistes en Afrique Occidentale, Paris, Errance/NEA, p. 261.

5 LINGANI Zakaria, 1988, La métallurgie du cuivre et des alliages cuivreux à Ouagadougou, mémoire de maîtrise, Université de Ouagadougou, IN.S. HU.S, Département d'Histoire et Archéologie, p.63.

6 https://fr.m.Wikipédia.org, dernière consultation le 22 janvier 2016.

7 LINGANI Zakaria, 1988, La métallurgie du cuivre et des alliages cuivreux à Ouagadougou, mémoire de maîtrise, Université de Ouagadougou, IN.S. HU.S, Département d'Histoire et Archéologie, p.61. pour l'achat de la plaque solaire, de la batterie et de la bobine électrique. L'entretien régulier des plaques solaires est fondamental pour éviter le dépôt de poussière qui diminue la production d'énergie.

\section{Conclusion}

Le progrès technique évolue dans le temps et l'espace, et l'Homme fait appel à sa créativité pour faire évoluer ses techniques en fonction de son environnement. La technique de fonte et de fabrication des marmites en aluminium exprime l'originalité de cette création. Le mécanisme crée par Adama Traoré dans le village de Kolonkan-Ba montre comment, dans un village, on peut utiliser des éléments modernes pour alléger le travail et obtenir de meilleurs rendements. Il dénote aussi de l'esprit inventif et les mutations continuelles dans les techniques.

8 LINGANI Zakaria, 1988, La métallurgie du cuivre et des alliages cuivreux à Ouagadougou, mémoire de maîtrise, Université de Ouagadougou, IN.S. HU.S, Département d'Histoire et Archéologie, p. 66.

9 CORREA DA SILVA Maclovia, 2010, « Être étudiant en Histoire des Techniques ", pp. 27-41, In Techniques, Patrimoine, Territoires de l'Industrie: Quel enseignement? International Workshop, 227 p., p. 33.

10 AL-ZOUBI Céline, 2008, L'agriculture tammari dans l'Atakora-Anthropo-histoire des techniques agricoles des Betammaribe de l'Atakora précolonial (Togo, Bénin) ; Pistes de recherche, Paris, Université de Paris 1-Panthéon-Sorbonne, mémoire en Histoire des Techniques, p. 84.

11 LINGANI Zakaria, 1988, La métallurgie du cuivre et des alliages cuivreux à Ouagadougou, mémoire de maîtrise, Université de Ouagadougou, IN.S. HU.S, Département d'Histoire et Archéologie, p.82.

12 CORNEVIN R, 1960, Histoires des peuples de l'Afrique noire, Boulogne-Billancourt, Berger Levrault, p. 650.

13 PAULME Denise, 1956, les sculptures de L'Afrique Noire, Paris, PUF, p. 26. 
14 LINGANI Zakaria, 1988, La métallurgie du cuivre et des alliages cuivreux à Ouagadougou, mémoire de maîtrise, Université de Ouagadougou, IN.S. HU.S, Département d'Histoire et Archéologie, p. 17.

15 ZOUNGRANA Ibrahim, 2015, L'artisanat du bronze d'art à Ouagadougou : un essai d'étude socioéconomique de 1915 à 201O, mémoire de maîtrise, Université de Ouagadougou, p. 43.
16 LINGANI Zakaria, 1988, La métallurgie du cuivre et des alliages cuivreux à Ouagadougou, mémoire de maîtrise, Université de Ouagadougou, IN.S. HU.S, Département d'Histoire et Archéologie, p. 83 . 


\section{Bibliographie}

AL-ZOUBI Céline, 2008, L'agriculture tammari dans l'Atakora-Anthropo-histoire des techniques agricoles des Betammaribe de l'Atakora précolonial (Togo, Bénin); Pistes de recherche, Paris, Université de Paris 1-Panthéon-Sorbonne mémoire en Histoire des Techniques.

CORNEVIN R., 1960, Histoires des peuples de l'Afrique noire, Boulogne-Billancourt, Berger Levrault, $715 \mathrm{p}$.

CORREA DA SILVA Maclovia, 2010, «Être étudiant en Histoire des Techniques ", pp. 27-41, In Techniques, Patrimoine, Territoires de l'Industrie : Quel enseignement? International Workshop, $227 \mathrm{p}$.

GARÇON Anne-Françoise et al., 2010, "Introduction », pp. IX-XVIII ; In Techniques, Patrimoine, Territoires de l'Industrie: Quel enseignement? International Workshop, $227 \mathrm{p}$.

GREBENART D., 1996, Les premiers métaux en Afrique de l'ouest, in la préhistoire de l'Afrique de l'ouest (nouvelles données sur la période récente), Nouakchott, Sépia.

GREBENART D., 1988, Les premiers métallurgistes en Afrique Occidentale, Paris, Errance/NEA, 289 p.

KIENON-KABORE Timpoko-Hélène, 2013, "L’imaginaire des techniques anciennes en Afrique subsaharienne », Communication au colloque sur "L’imaginaire et les techniques 》 du 14 au 16 novembre 2013, Paris, France (en cours de publication).

LINGANI Zakaria, 1988, La métallurgie du cuivre et des alliages cuivreux à Ouagadougou, mémoire de maîtrise, Université de Ouagadougou, IN.S. HU.S, Département d'Histoire et Archéologie, $173 \mathrm{p}$.
PAULME Denise, 1956, Les sculptures de L'Afrique Noire, Paris, PUF, $129 \mathrm{p}$.

ZOUNGRANA Ibrahim, 2015, L'artisanat $d u$ bronze d'art à Ouagadougou: un essai d'étude socioéconomique de 1915 à 2010, mémoire de maîtrise, Université de Ouagadougou, 140 p. 